\title{
Visualizing Excitement of Individuals and Groups
}

\author{
Kostiantyn Kucher \\ Linnaeus University \\ Växjo, Sweden \\ kostiantyn.kucher@lnu.se
}

\author{
Daniel Cernea \\ AGT International \\ Darmstadt, Germany \\ dcernea@agtinternational.com
}

\author{
Andreas Kerren \\ Linnaeus University \\ Växjo, Sweden \\ andreas.kerren@lnu.se
}

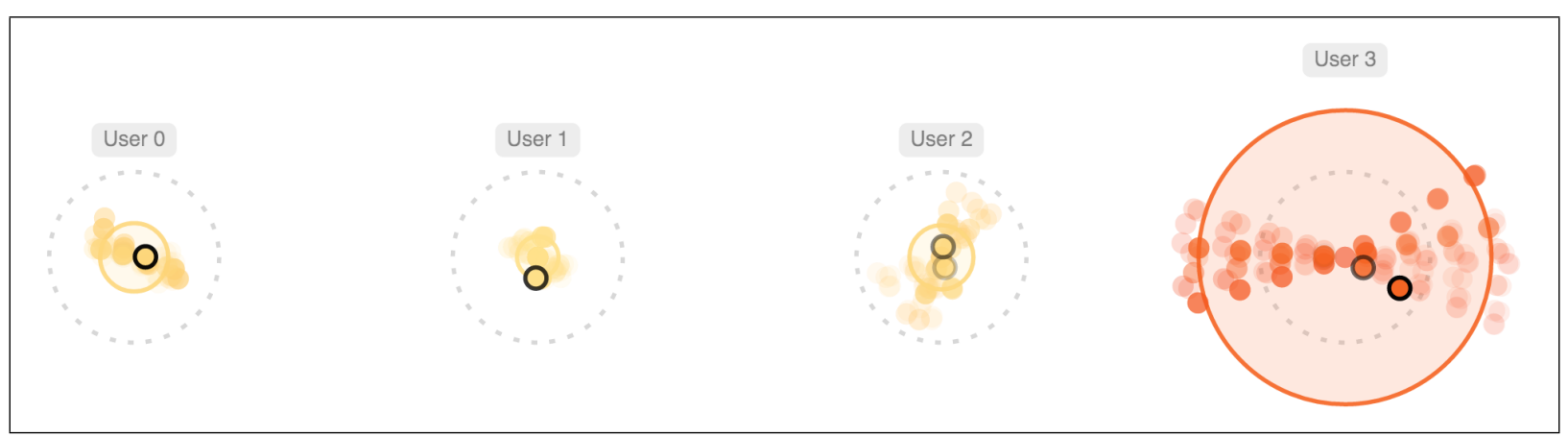

Figure 1: Overview of our visualization applied to a synthetic data set: each circular glyph corresponds to a single user, its concentric rings encode the baseline and current excitement values, and the nested dots provide the detailed information about time-varying measurement values. Here, the oscillating trail encoding is used for the nested dots.

\begin{abstract}
Excitement or arousal is one of the main emotional dimensions that affects our lives on a daily basis. We win a tennis match, watch a great movie, get into an argument with a colleague-all of these are instances when most of us experience excitement, yet we do not pay much attention to it. Today, there are few systems that capture our excitement levels and even fewer that actually promote awareness of our most exciting moments. In this paper, we propose a visualization concept for representing individual and group-level excitement for emotional self-awareness and group-level awareness. The data used for the visualization is obtained from smart wristbands worn by each of the users. The visualization uses animated glyphs to generate a real-time representation for each individual's excitement levels. We introduce two types of encodings for these glyphs: one focusing on capturing both the current excitement and the excitement history, as well as another focusing only on real-time values and previous peaks. The excitement levels are computed based on measurements of the user's galvanic skin response and accelerometer data from the wristbands, allowing for a classification of the excitement levels into experienced (excitement without physical manifestation) and manifested excitement. A dynamic clustering of the individual glyphs supports the scalability of our visualization, while at the same time offering an overview of the group-level excitement and its distribution. The results of a preliminary evaluation suggest that the visualization allows users to intuitively and accurately perceive both individual and group-level excitement.
\end{abstract}

EmoVis 2016 Workshop, March 10, 2016, Sonoma, CA, USA.

Workshop proceedings published by Linköping University Electronic Press.

Copyright (c) 2016 for this paper by its authors. Copying permitted for private and academic purposes.

\section{Author Keywords}

Excitement visualization; emotion visualization; group excitement; personal visualization; galvanic skin response.

\section{ACM Classification Keywords}

H.5.2 Information Interfaces and Presentation (e.g. HCI): User Interfaces; K.8.1 Personal Computing: Application Packages

\section{INTRODUCTION}

In every day of our lives, irrespective of the environment and activities we are involved in, we experience a multitude of different emotions that both affect and even guide our decision making. It comes then as no surprise that emotional theory and the measurement of human emotion has slipped further into focus in research and industry as a means to increase emotional awareness and devise emotion-adaptive computing systems.

In this context, user excitement or arousal is one of the main attributes used to capture emotional variation in various dimensional models [24, 28] from emotion theory. Throughout our daily lives, we experience varying levels of excitement in a multitude of settings: calmness during our commute to work, stress during a business meeting, excitement while watching sports, etc. Still, there are few solutions that try to capture and represent user excitement in the context of emotional self-awareness and group-level awareness. Imagine if you could employ a visualization system in order to explore your most stressful moments of the day, in order to improve your habits and remove unwanted stimuli. Similarly, in terms of group-level awareness, consider as an example a visualization that shows your and your friends' real-time excitement levels while playing a multi-player strategy game. 
In this paper, we introduce a novel concept for visualizing individual and group-level excitement in real-time based on the information streamed from smart wristbands worn by each member of the group. The data is gathered from a set of Microsoft Band 2 wearables [25] that periodically measure the galvanic skin response (GSR) and accelerometer (ACC) values for each user. We process this data and compute the levels of experienced excitement (excitement that is detected through the GSR measurements but is not manifested physically) and manifested excitement (high GSR levels coupled with sudden motion of the arms, e.g., cheering at a soccer game).

The visualization is based on animated glyphs that encode each person's excitement levels and allow them to perceive the overall excitement of the group. Figure 1 presents the excitement levels for four individuals. We propose two visual encodings for the glyph: on the one hand, focusing on the representation of the current and previous levels of excitement for each individual, while on the other hand, with scalability in mind, employing animation to better convey the current level of excitement for each person. A dynamic layout enables glyphs with similar excitement levels to be grouped in order to better perceive the distribution of the excitement levels in large groups.

In the following sections, we will initially discuss related research in terms of emotional theory, emotion measurement, and personal visualization. We continue by highlighting the data acquisition and processing for the excitement levels of the individuals. Next, we detail the visualization design and its implementation, followed by a preliminary evaluation of our concept. We conclude by presenting our final remarks and future steps.

\section{RELATED WORK}

Nowadays, there is a set of different techniques that are being used for estimating user emotions, ranging from classification of facial expressions and voice tonality to neurological activity and other physiological measurements. While these techniques all have their various estimation capabilities in the context of emotional theory, when focusing on the dimension of arousal some of the most accurate predictors are based on biosignals such as heart rate, respiration, electrodermal activity, body temperature, pupil size variation, all affected by the autonomic nervous system (ANS) [31].

Throughout the decades, electrodermal activity (EDA) or galvanic skin response (GSR) has shown promising results in terms of correlation with user excitement levels [26, 27, 32]. In this technique, two electrode measure the skin conductivity of a user. The conductivity is affected by the moisture that is generated by the eccrine sweat glands, which can be found in our hands and feet, and are mostly responsive to changes in emotional arousal. The activation of these sweat glands is not under the conscious control of a person and can be tied especially to a heightened level of arousal [35] and cognitive workload [30].

Recently, smart wristbands were introduced on the market featuring GSR sensors (e.g., Microsoft Band 2 [25], Empatica
E4 [13]), enabling a new dimension of personal data collection and interpretation. Equipped with additional sensors like accelerometers, these wearables have the potential to bring emotion estimation to the real world by considering the context and the activity of the users when processing their physiological signals [17].

While many techniques based on physiological measures have already been used to estimate excitement levels in individuals, there are few systems that focused on raising awareness and visualizing these emotional states. Part of the area of personal visualization $[18,33]$, emotion visualization is one of the approaches used to inspect and interpret information about user and group affective states.

McDuff et al. [23] introduced AffectAura, a visualization for estimated emotional states for a single user over time using data from multiple sensors. Their work uses an aggregated visual representation for representing valence, arousal and engagement levels for a longer time span of one day. In contrast, our approach is focused on the real-time visualization of a single attribute (excitement) for short time spans, and supports measurements from multiple users simultaneously.

In terms of real-time visualization of emotions, Cernea et al. [7] introduced Emotion-prints, a visualization system for touch-enabled interfaces, where the current level of a user's valence and arousal (estimated through EEG measurements) is represented in the shape of a animated halo around the virtual objects that the user touches. Saari et al. [29] introduced a mobile emotion visualization system devised for improving group performance and awareness. One step further, the GAT visualization [5] enabled the representation of consistent affective experiences at a group level in real-time, thus enabling emotional awareness in teams and improving collaboration. However, emotions have been visualized through a wide range of representations, ranging from expressioninspired approaches-avatars and icons [16, 19, 22]—and to abstract interface integrations $[6,16]$.

In the context of group psychology, emotional awareness has been linked to decision making [15] and communication [8, 12], while emotional engagement (including excitement) has been shown to be closely linked to gamification [21].

Visualization of real-time measurements collected from multiple wristband devices presents additional challenges and can be interpreted as streaming data visualization. One of the earliest papers on this topic is a work by Wong et al. [36] who defined the corresponding visualization challenges: the data may arrive as an unpredictable and unbounded stream without any clear patterns. The authors proposed several techniques to visualize streaming data with scatterplots using variations of multidimensional scaling (MDS) [4]. Alsakran et al. [2] introduced STREAMIT, a scalable visualization system that uses a force-direct dynamic system to lay out visual items representing text documents. Huron et al. [20] discussed $\mathrm{Vi}$ sual Sedimentation, a powerful metaphor that uses animation to represent aging and aggregation of incoming data entries. In contrast to these papers, our work does not focus specifically on the streaming nature of data (i.e., it is equally ap- 
plicable to non-streaming data sets), but takes this aspect into account when providing a personal visualization.

Liu et al. [22] described an emotion recognition and visualization technique based on EEG measurements. They supported processing of real-time data at $128 \mathrm{~Hz}$ with a sliding window algorithm and used a 3D avatar for encoding of emotions for a single user. In the case of our approach, data measurements are made at a much lower frequency and the supported visual encodings are much more compact. Furthermore, our work supports visualization of excitement for multiple users simultaneously.

Finally, Cottam et al. [10] proposed a taxonomy for streaming data visualization techniques based on dynamics of spatial and retinal variables. They introduced design guidelines based on the comparison task, stability of the displayed items set, and effects of time-varying data attributes. In our case, a single design option based on their guidelines is not sufficient since we want to provide a visualization of streaming data items grouped by user-i.e., there are two representation levels-with support for fixed and dynamic layouts at the inter-user level and two different visual encodings at the intra-user level.

\section{DATA}

We have used Microsoft Band 2 devices [25] to record galvanic skin response (GSR) and accelerometer (ACC) values for several people engaged in common activities. The activities involved three to four individuals and included: watching a movie, an opera, and going to a museum. During these activities, each person was wearing a device that would provide GSR and ACC measurements at approximately 0.2 and 9-11 $\mathrm{Hz}$, respectively. This data from each Band 2 was streamed in real-time to an Android mobile phone through a bluetooth connection, from where it was forwarded and stored in a secured database. Currently, our visualization system can access only text-based exports from this database, with future version being able to obtain information directly from the server.

For our purposes, ACC values were sampled to synchronize them with GSR values. For the GSR values, a normalization was applied based on the extracted normal and stressed states, as described in [3]. These states have been extracted either offline, or based on a calibration stage where the intervals for the normal and stressed states were estimated.

Furthermore, the GSR values can fluctuate due to user physical activity or-as perceived in our measurements - a tight fit of the devices on the wrist leading to excessive sweating. To compensate for these events, the ACC values from the mobile device were compared with the ones from the Band 2s. If the user was exerting physical activity as detected both by the mobile device and the wristband, an increased level of excitement was not reported. Moreover, as slow constant sweating could increase the GSR values over time, a sliding window was applied that enables the detection of local increases in the GSR values, thus capturing acute states of excitement.

The resulting data sets comprised timestamped recordings of two variables, normalized in range $[0 ; 1]$ for a correspond-

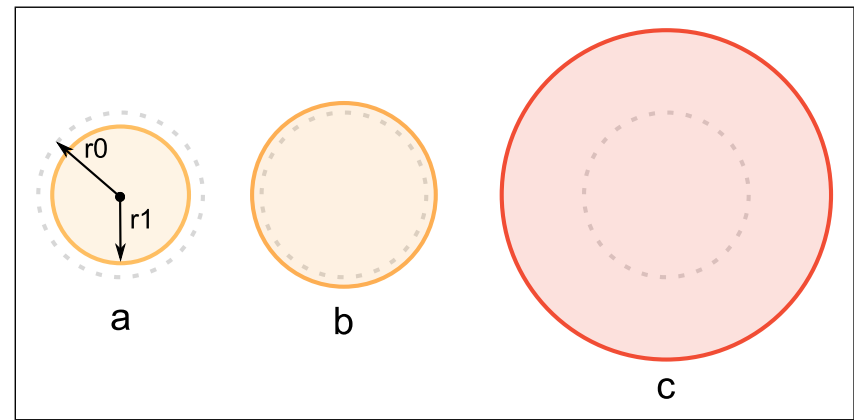

Figure 2: Design of a glyph: (a) a case with rather small GSR and ACC values and highlighted radii; (b) a case with a large GSR and small ACC value; (c) a case with both large GSR and ACC values.

ing user, that are expected to be emitted every 5 seconds for sessions ranging from 20 minutes to several hours. However, there is a degree of uncertainty caused by measurement delays and errors (e.g., the wearables can lose the contact with the user's arm during a measurement session) as well as potential networking delays when transferring data to the visualization front-end (which is less of a concern for pre-recorded data). These issues were relevant factors for our visualization design, as the visualization should support streaming data in order to enable real-time measurements.

Additionally, several synthetic data sets were created for development and demonstration purposes that use a random walk model for each user's GSR and ACC values. Such data sets are a viable option to test the approach with a larger number of users (for instance, 10-15), as the real devices can be unavailable in such quantity.

\section{VISUALIZATION TASKS AND DESIGN}

We have had the following visualization tasks in mind for our work:

- monitoring of current excitement values for each user in a group,

- temporal overview of excitement values (for relatively short periods of time) for each user, and

- clustering based on excitement value similarity between users.

Our overall visualization design has two levels: the intra-user level for visualizing measurements for an individual user, and the inter-user level for visualizing measurements for a group of users simultaneously.

We represent the data for each user as a circular glyph, thus enabling a scalable visualization that can capture the excitement levels for small to medium sized groups of people. Each glyph is presented with two groups of elements: concentric circles (rings) and trails. Concentric circles displayed in Figure 2(a) encode the baseline GSR value boundary (dashed circle with radius $r O$ ) and the combination of GSR and ACC values (shaded circle with radius $r 1$ ). The motivation for this encoding was the assumption that GSR values correspond to 


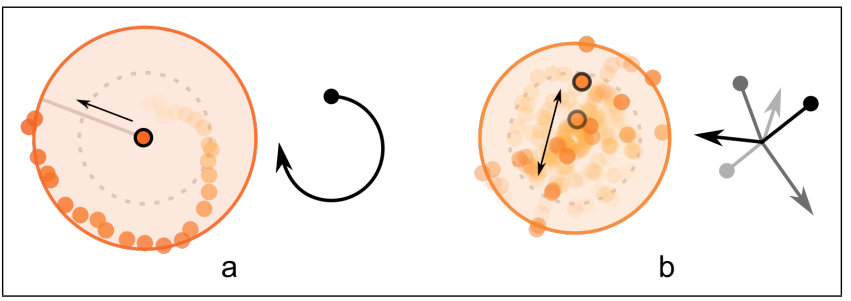

Figure 3: Design of trails nested in a glyph: (a) a clock-style trail with angle increasing over time; (b) an oscillating trail with angle changing randomly. In both encodings, the target distance from the glyph center encodes the combined GSR + ACC values.

experienced excitement levels, while ACC values correspond to manifested excitement levels (e.g., the behaviour of a person clapping hands while cheering for a soccer team would be easily observable this way). ACC values are on average larger than the corresponding GSR values in our recorded data sets. Therefore, we use the GSR value as the base, but if it reaches a certain threshold (set to 0.8 by default), the ACC value is added to it to produce the combined excitement level. For example, the radius of the shaded circle that barely exceeds the baseline value would signal that a user is excited based mostly on GSR (Figure 2(b)), but if ACC values are also large, the radius of the shaded circle (and the glyph in general) would exceed the baseline level significantly (Figure 2(c)), suggesting a manifested excitement.

To provide a temporal overview for the combined GSR + ACC values for each user, our technique supports two visual encodings based on animation: clock-style and oscillating trails which are rendered for each incoming data entry. The clock-style encoding demonstrated in Figure 3(a) uses a clock metaphor and assigns increasing angle values for items corresponding to data entries (to complete the metaphor, a clock hand is rendered as well). With this representation, a data entry is mapped to a single visual item (dot), its timestamp is mapped to the angle value, and its combined excitement value is mapped to the distance from glyph center. A new item is created for any incoming entry, highlighted with a thick stroke, and its transition from glyph center to the target position is animated to help the visualization users focus on this latest entry. Opacity of the visual items decays over time, and with the default settings, items fade out completely in a given time interval, which is also the default setting for a single "clock" rotation period. This time interval can be set in the configuration of the visualization to better suit the scenario in which it is employed. As such, the clock-style encoding is designed to provide a rather precise and uncluttered overview of the values for a limited time period and facilitates comparison between several glyphs.

The second visual encoding demonstrated in Figure 3(b) follows an oscillation metaphor suggestive of the user's current excitement level. For each incoming data entry, a number of dots is created in succession, the latest created dot is highlighted with a thick stroke, and the overall effect of the animation resembles a trajectory rendering. The excitement value is used to limit the distance from the glyph center for these trails, thus resulting in an oscillatory motion inside the boundary of the excitement circle. In order to better capture the user's excitement levels, the oscillation speed can increase with the level of excitement, resulting in faster motion of the dots for higher levels of excitement (i.e., larger excitement circles). This correlation of the excitement levels and oscillation speed has also shown initial positive results in terms of perceiving the group-level excitement in larger groups (12 or more individuals). The opacity of the dots quickly decays over time, but the items do not fade out completely with the default settings. Furthermore, the movement angle changes randomly when passing through the glyph center, in order to ensure an even distribution of the dot trails over the entire surface of the representation. This way, the visualization users can perceive the maximum excitement values over a time period rather than the excitement history of the user.

The excitement values (i.e., the combined GSR + ACC values) are used for the color coding of both general glyph elements and trail elements. We support a number of color maps from ColorBrewer [9] for three sequential data classes, and use the corresponding colors for the excitement values of 0.0 , 1.0, and 2.0 (since we expected both GSR and ACC values to be in range $[0 ; 1])$. The colors for 0.0 and 1.0 are then interpolated using the HCL model for an actual excitement value (values 1.0 and 2.0 are used if the value exceeds 1.0). For instance, with the default settings the values $0.0,1.0$, and 2.0 are mapped to yellow, orange, and red, respectively. The colors are used by the outer shaded glyph circles as well as the trail dots.

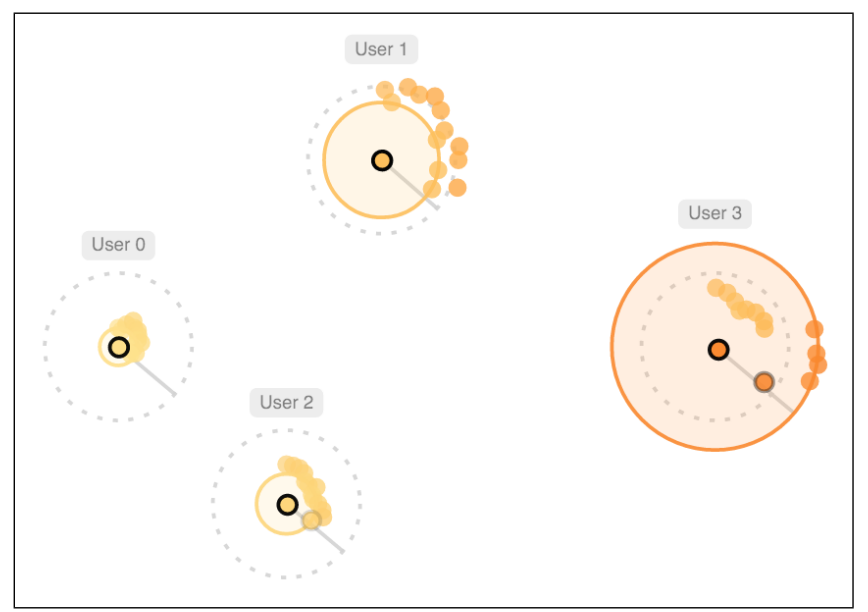

Figure 4: The displayed force-based layout for glyphs is based on similarity of latest excitement values between users.

The final aspect of our technique is the positioning of the glyphs. We support two approaches that could both be described as derived data-driven placement strategies following the Ward's taxonomy [34]. By default, glyphs are placed on a uniform grid based on the corresponding user ID values (see Figure 6 for an example). This approach may be useful when monitoring a specific glyph or continuously comparing values for several glyphs. The users can also enable a dynamic force-based layout [14] that is updated on every 
incoming data entry. In this case, the expected distances between glyphs are set to be proportional to differences between the latest corresponding GSR + ACC values. This approach facilitates monitoring the group dynamics and identification of clusters. Figure 4 demonstrates how users can be grouped, User 3 being overexcited while Users 0 and 2 experience low excitement.

\section{IMPLEMENTATION DETAILS}

Our prototype is implemented as a static web page with JavaScript code that uses D3 [11] for layout and rendering. The design and implementation of the prototype support streaming data. Currently, we provide a choice of several recorded or synthetic data sets with multiple users. The implementation "replays" the selected data set and emits JSON entries for single measurements based on the recorded timestamps.

The actual visualization code processes incoming entries in a streaming fashion. It is designed to handle data for user IDs not encountered previously, to update the global layout, and to interrupt current animations at the intra-user level. Figure 5 provides an overview of the update algorithm: a new glyph for a user is rendered if necessary, the layout is updated, and finally, the new data item is rendered with regard to the currently selected visual encoding for trails.

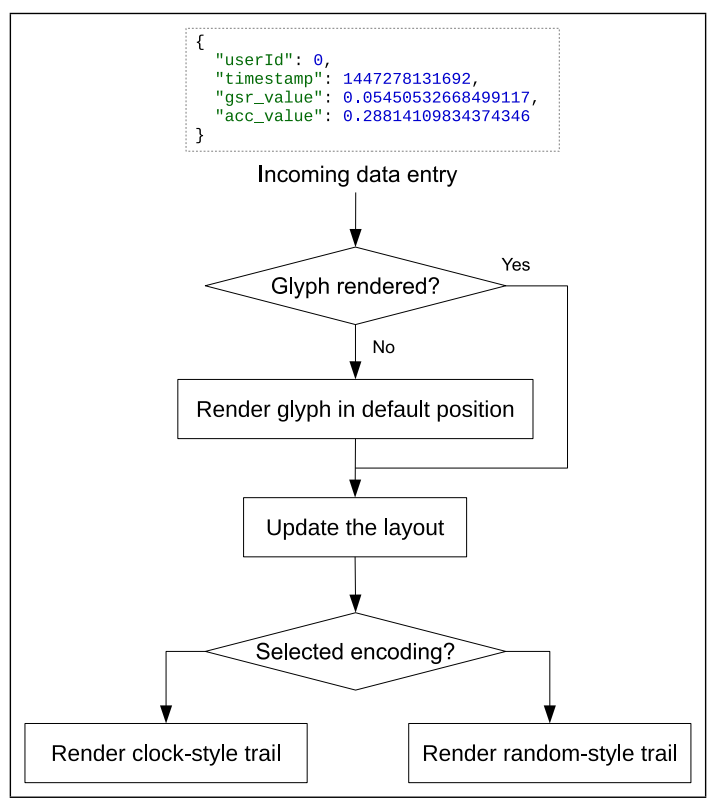

Figure 5: Diagram of the update algorithm for incoming data entries.

Figure 6 demonstrates the interface of our visualization prototype. Users are provided with options to change the visual encoding (in this case, the intra-user visualizations are reset) as well as to modify multiple visualization parameters-e.g., visual encodings and layout of the glyphs, both visual encodings for trails, etc. Moreover, the user can change the color map, the visibility of the glyph boundary elements, or adjust

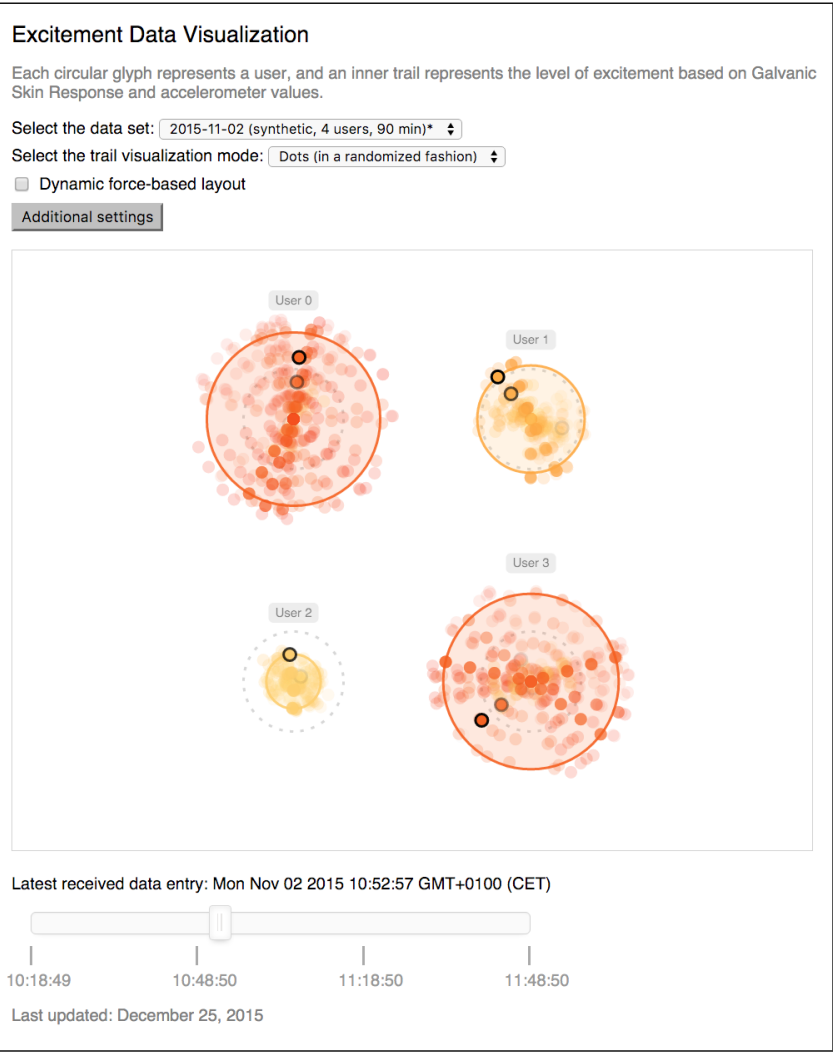

Figure 6: Screenshot of the prototype interface taken for a synthetic data set.

the animation durations and opacity values for trails. To facilitate exploration, the visualization also supports zoom \& pan.

We also included a temporal slider to support navigation for our pre-recorded data sets. This choice was made mostly for the prototype testing purposes-as stated above, our general goal is to support actual streaming data sources which would make temporal navigation limited with regard to the current point in time.

\section{VALIDATION AND DISCUSSION}

A preliminary evaluation has been executed in order to validate our concept. The question we wanted to answer was whether the excitement visualization design we proposed was a suitable visual representation that could satisfy all three requirements discussed above. A group of 8 participants (5 male, 3 female, with ages between 25 and 33) were given the opportunity to visually inspect the excitement levels for up to 4 different users at the same time. Based on their experience with the visualization, the participants were asked to fill out a questionnaire.

The questionnaire included both closed-ended and openended questions. The closed-ended questions were scaled questions distributed on a 5-point Likert scale, where 1 represented "disagree" and 5 represented "agree". 


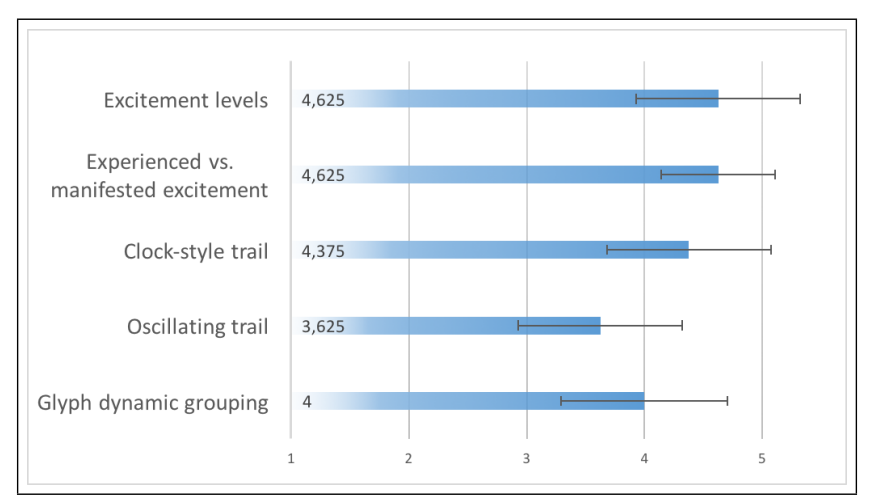

Figure 7: Averages and standard deviations for 5-point Likert scale answers provided by the questionnaire participants.

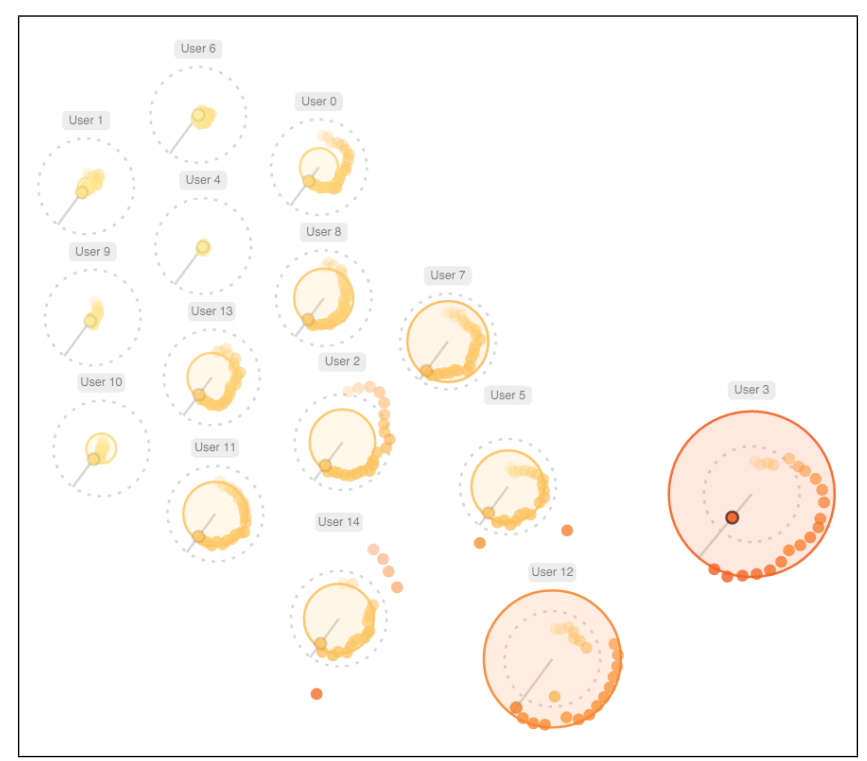

Figure 8: Visualization of a synthetic data set with 15 users with the dynamic force-based layout.

The results of the questionnaire suggest an overall positive and accurate perception of the proposed excitement visualization (see Figure 7). On average, participants rated their ability to differentiate between the various excitement levels in the visualization at 4.6. Given a concrete example (similar to Figure 4), they were asked how many people of the four individuals in the visualization were excited and manifesting it, all but one of the participants gave the correct answer, further supporting the idea that our representation is intuitive and easy to perceive. Moreover, participants felt confident about differentiating between experienced and manifested excitement (average of 4.6).

When asked about the perception of the history of excitement for each individual, the clock-style trail offered a clearer perception of the excitement history (with an average of 4.4) compared to the oscillating trail (average of 3.6). However, as previously stated, the oscillating trails are not meant to encode the excitement history per se, but rather capture the maximum excitement level experienced in a given time period. This has been also reflected in the comments, as one of the participants stated that the oscillating trail "could surface peak events". In terms of group-level excitement, the questionnaire participants found that the grouping of the circles usually helped them to accurately convey the overall excitement level in the group (average of 4.0).

In terms of open-ended questions, the participants had positive remarks related to the visual design, its simplicity and the animation elements that further support the correct perception of the excitement levels. When looking at the issues, participants suggested that the visualization can be difficult to read at times, especially as the grey circle of each individual is not always clearly visible. Additionally, multiple participants suggested extending the visualization to also incorporate positive and negative emotions (emotional valence) in the representation.

We have also inspected the excitement data we have gathered during the recording sessions (at the movies, opera, and museum) in order to investigate our visualization's capability to correctly convey moments of peak excitement. A comparison was executed between the peak excitement moments in the visualization and the self-reported logs of the participants. In multiple instances, we could observe a correlation between the self-reported and visualized excitement levels, in the case of both homogeneous group excitement (all or most members of the groups got excited) and individual excitement (only one member of the group got excited-Figure 9). During both the movie and opera activities, the participants had similar levels of base excitement. However, at certain captivating or emotional moments, the excitement levels increased synchronously. At the same time, the data obtained from the museum had fewer extracted peaks, partly due to the additional noise generated by the users walking, but also due to the fewer high excitement peaks-fact confirmed post-task by the participants as well.

While we had a rather small number of simultaneous users wearing smart wristbands in the recording sessions, the proposed visualization does scale to larger numbers of users. A visualization of a synthetic data set with 15 users in Figure 8 demonstrates that the grouping of users by excitement is present and quite easy to perceive, even though the amount of detailed information about each individual user may be large and harder to follow as the glyph changes its position with the dynamic layout. The relatively large number of users may also make the force-based layout unstable, which we plan to address in our future work.

We have also analyzed the aspects of our visualization technique with regard to the taxonomy by Cottam et al. for streaming data visualization [10]. At the inter-user level, we expect new entries (i.e., glyphs) to be added dynamically, which corresponds to the Create category with regard to spatial dimension (this applies to both supported layouts). Incoming data entries affect the attributes of concentric circles for a known range of values, which corresponds to the Known Scale category with regard to retinal dimension. According to Cottam et al., this combination of categories can be 


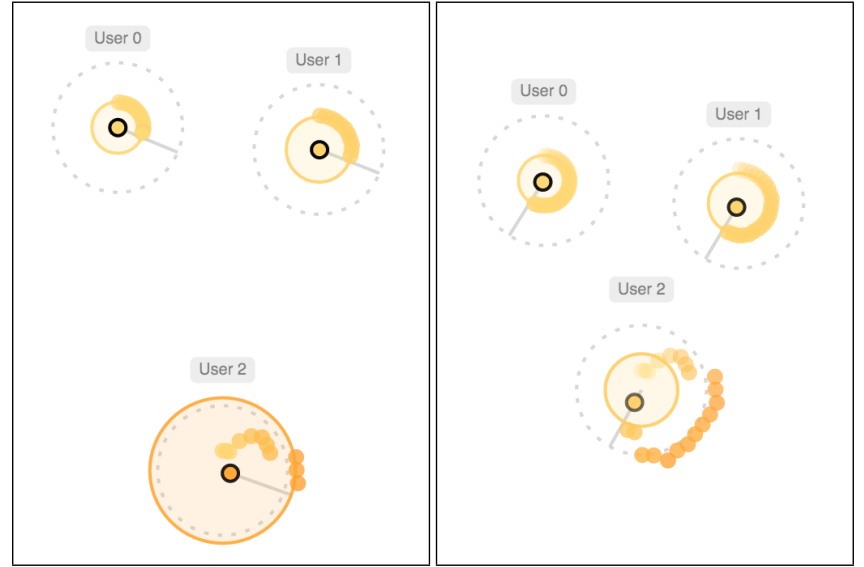

Figure 9: Two screenshots of the excitement visualization of the museum dataset. User 2, a hobby mechanic, noticed a classical car he only knew from images. This, combined with him waving to the other two ("Come and see this!") increased his level of excitement (left). After some time, his level of excitement decreased again to normal (right). As visible in the visualization, the other two individuals were rather unimpressed by the vehicle.

classified as transitional with regard to the elements' identity preservation. Such techniques usually favor comparison over short time spans, and this statement holds for our approach if only glyph layout and concentric circle elements are considered. However, our visual encodings for trails provide an overview for longer time spans than the elements mentioned above (even though the clock-style and oscillating trails on their own would be classified as Create \& Delete $\times$ Known Scale and Create $\times$ Known Scale, respectively).

\section{CONCLUSIONS AND FUTURE WORK}

In this paper, we have introduced a novel visualization for representing real-time and historical excitement levels for individuals and groups. Two different visual encodings are proposed for representing individual excitement levels through animated glyphs: one focusing on capturing real-time and previous excitement levels, and one more suited for capturing individual excitement peaks and the distribution of excitement in larger groups. The perception of the group-level excitement is aided by a dynamic layout that clusters glyphs with similar excitement levels. An initial evaluation of our visualization offered promising results as well as a couple of potential improvements in terms of visual complexity.

We see several opportunities for the future work:

- The current visual encodings are limited with regard to a temporal overview for longer periods of time (such as 30 minutes or longer). This could be alleviated by introducing an additional time-varying representation [1] either attached to user-related glyphs, or a completely separate one (e.g., a simple line plot). In the latter case, an additional representation based on aggregated values could also be used to provide an overview for the whole group of users.
- Finally, the dynamic layout for user-related glyphs could be improved by either modifying the current implementation (e.g., the initial positions for the dynamic layout could be based on MDS results [4]), or changing the force-based model to use an explicit clustering algorithm.

\section{ACKNOWLEDGMENTS}

This work was partly funded by the framework grant "The Digitized Society-Past, Present, and Future" from the Swedish Research Council (Vetenskapsrådet) [grant number 2012-5659].

\section{REFERENCES}

1. Aigner, W., Miksch, S., Schumann, H., and Tominski, C. Visualization of time-oriented data. Springer Science \& Business Media, 2011.

2. Alsakran, J., Chen, Y., Zhao, Y., Yang, J., and Luo, D. STREAMIT: Dynamic visualization and interactive exploration of text streams. In Proceedings of the IEEE Pacific Visualization Symposium (PacificVis), IEEE (2011), 131-138.

3. Bakker, J., Pechenizkiy, M., and Sidorova, N. What's your current stress level? Detection of stress patterns from GSR sensor data. In Proceedings of the 2011 IEEE 11th International Conference on Data Mining Workshops (ICDMW'11), IEEE (2011), 573-580.

4. Borg, I., and Groenen, P. J. F. Modern multidimensional scaling: Theory and applications. Springer Science \& Business Media, 2005.

5. Cernea, D., Ebert, A., and Kerren, A. Visualizing group affective tone in collaborative scenarios. In Poster Abstracts of the Eurographics Conference on Visualization (EuroVis '14) (2014).

6. Cernea, D., Weber, C., Ebert, A., and Kerren, A. Emotion Scents - a method of representing user emotions on GUI widgets. In Proceedings of the SPIE 2013 Conference on Visualization and Data Analysis (VDA '13), vol. 8654, IS\&T/SPIE (2013).

7. Cernea, D., Weber, C., Ebert, A., and Kerren, A. Emotion-prints: Interaction-driven emotion visualization on multi-touch interfaces. In Proceedings of the SPIE 2015 Conference on Visualization and Data Analysis (VDA '15), vol. 9397, IS\&T/SPIE (2015).

8. Charlton, B. Evolution and the cognitive neuroscience of awareness, consciousness and language. In Psychiatry and the Human Condition. Radcliffe Medical Press, 2000.

9. ColorBrewer 2.0 — color advice for cartography. http: //colorbrewer2 .org/. Accessed January 28, 2016.

10. Cottam, J. A., Lumsdaine, A., and Weaver, C. Watch this: A taxonomy for dynamic data visualization. In Proceedings of the IEEE Conference on Visual Analytics Science and Technology (VAST) (2012), 193-202. 
11. D3-data-driven documents. http://d3js.org/. Accessed January 28, 2016.

12. Druskat, V. U., and Wolff, S. B. Building the emotional intelligence of groups. Harvard Business Review 79, 3 (2001), 80-90.

13. Empatica E4 Wristband. https : //www. empatica.com/e4-wristband. Accessed January 28, 2016.

14. Fruchterman, T. M. J., and Reingold, E. M. Graph drawing by force-directed placement. Software: Practice and Experience 21, 11 (1991), 1129-1164.

15. García, O., Favela, J., Licea, G., and Machorro, R. Extending a collaborative architecture to support emotional awareness. In Proceedings of the Workshop on Emotion Based Agent Architectures (EBAA '99) (1999), 46-52.

16. García, O., Favela, J., and Machorro, R. Emotional awareness in collaborative systems. In Proceedings of the International Workshop on Groupware (CRIWG '99), IEEE (1999), 296-303.

17. Healey, J. A. Affect detection in the real world: Recording and processing physiological signals. In Proceedings of the 3rd International Conference on Affective Computing and Intelligent Interaction and Workshops (ACII) (2009).

18. Huang, D., Tory, M., Aseniero, B. A., Bartram, L., Bateman, S., Carpendale, S., Tang, A., and Woodbury, R. Personal visualization and personal visual analytics. IEEE Transactions on Visualization and Computer Graphics 21, 3 (2015), 420-433.

19. Huisman, G., van Hout, M., van Dijk, E., van der Geest, T., and Heylen, D. LEMtool: measuring emotions in visual interfaces. In Proceedings of the SIGCHI Conference on Human Factors in Computing Systems (CHI '13), ACM (2013), 351-360.

20. Huron, S., Vuillemot, R., and Fekete, J.-D. Visual sedimentation. IEEE Transactions on Visualization and Computer Graphics 19, 12 (2013), 2446-2455.

21. Kapp, K. M. The Gamification of Learning and Instruction: Game-based Methods and Strategies for Training and Education. Pfeiffer, 2012.

22. Liu, Y., Sourina, O., and Nguyen, M. K. Real-time EEG-based human emotion recognition and visualization. In Proceedings of the International Conference on Cyberworlds (CW), IEEE (2010), 262-269.

23. McDuff, D., Karlson, A., Kapoor, A., Roseway, A., and Czerwinski, M. AffectAura: An intelligent system for emotional memory. In Proceedings of the SIGCHI Conference on Human Factors in Computing Systems (CHI '12), ACM (2012), 849-858.

24. Mehrabian, A. Basic Dimensions for a General Psychological Theory: Implications for Personality,
Social, Environmental, and Developmental Studies. Social Environmental and Developmental Studies. Oelgeschlager, Gunn \& Hain, 1980.

25. Microsoft Band. https://www.microsoft. com/microsoft-band/en-us. Accessed January 28, 2016.

26. Nakasone, A., Prendinger, H., and Ishizuka, M. Emotion recognition from electromyography and skin conductance. In Proceedings of the 5th International Workshop on Biosignal Interpretation (BSI-05) (2005), 219-222.

27. Poh, M.-Z., Swenson, N. C., and Picard, R. W. A wearable sensor for unobtrusive, long-term assessment of electrodermal activity. IEEE Transactions on Biomedical Engineering 57, 5 (2010), 1243-1252.

28. Russell, J. A. A circumplex model of affect. Journal of Personality and Social Psychology 39, 6 (1980), 1161.

29. Saari, T., Kallinen, K., Salminen, M., Ravaja, N., and Yanev, K. A mobile system and application for facilitating emotional awareness in knowledge work teams. In Proceedings of the 41st Annual Hawaii International Conference on System Sciences (HICSS '08) (2008), 44-53.

30. Shi, Y., Ruiz, N., Taib, R., Choi, E., and Chen, F. Galvanic skin response (GSR) as an index of cognitive load. In CHI '07 Extended Abstracts on Human Factors in Computing Systems (CHI EA '07), ACM (2007), 2651-2656.

31. Valenza, G., and Scilingo, E. P. Autonomic Nervous System Dynamics for Mood and Emotional-State Recognition. Springer, 2014.

32. Villarejo, M. V., Zapirain, B. G., and Zorrilla, A. M. A stress sensor based on galvanic skin response (GSR) controlled by ZigBee. Sensors 12, 5 (2012), 6075-6101.

33. Wang, S., Tanahashi, Y., Leaf, N., and Ma, K.-L. Design and effects of personal visualizations. IEEE Computer Graphics and Applications 35, 4 (2015), 82-93.

34. Ward, M. O. A taxonomy of glyph placement strategies for multidimensional data visualization. Information Visualization 1, 3-4 (2002), 194-210.

35. Westerink, J. H. D. M., van den Broek, E. L., Schut, M. H., van Herk, J., and Tuinenbreijer, K. Computing emotion awareness through galvanic skin response and facial electromyography. In Probing Experience, J. H. D. M. Westerink, M. Ouwerkerk, T. J. M. Overbeek, W. F. Pasveer, and B. de Ruyter, Eds., vol. 8 of Philips Research. Springer, 2008, 149-162.

36. Wong, P. C., Foote, H., Adams, D., Cowley, W., and Thomas, J. Dynamic visualization of transient data streams. In Proceedings of the IEEE Symposium on Information Visualization (INFOVIS '03), IEEE (2003), 97-104. 